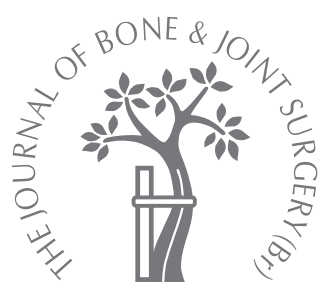

R. Scagnelli, G. Bianco,

D. Imarisio

From Ospedale Civile di Saluzzo, Saluzzo, Italy

R. Scagnelli, MD Orthopaedic Surgeon

- G. Bianco, MD, Orthopaedic

Surgeon

D. Imarisio, MD, Orthopaedic

Surgeon

Department of Orthopaedics

and Traumatology

Ospedale Civile di Saluzzo, Via

Spielberg 58, 12037 Saluzzo,

Italy.

Correspondence should be sent to $\mathrm{Dr} \mathrm{D}$. Imarisio; e-mail:

d.imarisio@libero.it

(C)2009 British Editorial Society

of Bone and Joint Surgery

doi:10.1302/0301-620X.91B7.

$22315 \$ 2.00$

$J$ Bone Joint Surg [Br] 2009;91-B:968-71.

Received 16 January 2009;

Accepted after revision 19

March 2009

- CASE REPORT

\title{
Cadaver bone-tendon graft for xanthomatosis of the tendo Achillis
}

\begin{abstract}
We describe a 63-year-old man who had xanthomatosis of the right tendo Achillis. He had undergone excision of the left tendo Achillis 17 years earlier without reconstruction for the same condition. The neurological history and examination were normal. Blood investigations showed hypercholestrolaemia, for which he was being treated with statins. He was referred with pain in the right tendo Achillis and problems with footwear.

He was treated by excision of the right tendo Achillis, the xanthomatous nodules and the involved skin, followed by reconstruction with a cadaver bone-tendon graft. At follow-up eight months post-operatively, the scar had healed well. He walked without pain and could wear any type of shoe. Plain radiographs showed that the bone graft had healed. The American Orthopaedic Foot and Ankle Society hindfoot score was 95/100. The patient's subjective evaluation of the result was very good.
\end{abstract}

Xanthomas are composed of lipid-laden histocytes. They appear as yellow patches, papules or nodules in the skin, tendons and soft tissues. Bilateral involvement of the tendo Achillis has been reported and may be associated with high levels of plasma lipids. ${ }^{1}$ This has also been described in cerebrotendinous xanthomatosis, a rare lipid-storage disease caused by a mutation in the sterol 27-hydroxylase gene (CYP 27). ${ }^{2,3}$ The condition usually appears in the first 30 years of life but the clinical presentation can be very variable with neurological symptoms (ataxia, depression, epilepsy and mental deterioration), cataract, atherosclerosis and tendinous xanthomas. Xanthomas in the tendo Achillis can cause pain, swelling, loss of function and problems with footwear. A number of procedures have been described for the reconstruction of the tendon after excision for chronic disease. The use of a local tendon transfer such as flexor hallucis longus (FHL) or peroneus brevis has been suggested, ${ }^{4,5}$ but other studies have reported the use of a free autologous tendon graft, such as gracilis, semitendinosus or fascia lata. ${ }^{6}$ The use of a cadaver allograft of tendo Achillis has also been advocated. ${ }^{7}$

\section{Case report}

A 63-year-old man presented with large mildly painful nodules in the right tendo Achillis. The skin was discoloured but not ulcerated. He had problems with wearing any type of shoe because of the nodules (Fig. 1). He also had asymptomatic xanthomas affecting the knees and the right elbow. There was no family history of xanthomatosis and neurological examination was normal. Blood investigations showed hypercholestrolaemia with a serum level of $240 \mathrm{mg} / \mathrm{dl}$ (normal 40 to $50 \mathrm{mg} / \mathrm{dl}$ ) and he had been taking atorvastatine $10 \mathrm{mg} / \mathrm{day}$ for ten years. He had undergone surgery 17 years earlier for a similar problem in the left tendo Achillis, which had been excised with no attempt at reconstruction (Fig. 1). Histological examination had confirmed the diagnosis of xanthomatosis.

MRI of the right tendo Achillis showed alteration of the structure of the tendon and the surrounding soft tissues (Fig. 2).

Operative technique. The tendo Achillis, xanthomatous nodules and surrounding involved skin were removed completely. The excised tendon was grossly deformed, thicker than normal, with inelastic fibrotic tissue replacing the normal tendinous tissue (Fig. 3). Reconstruction was undertaken using a frozen cadaver bone-tendon graft of tendo Achillis from the bank of musculoskeletal tissues of the CTO Hospital in Turin, Italy. The graft had been sterilised by gamma irradiation and frozen at $-80^{\circ} \mathrm{C}$.

The tendon was resected up to the musculotendinous junction where the muscle appeared macroscopically healthy. An osteotomy of the posterior apophysis of the calca- 


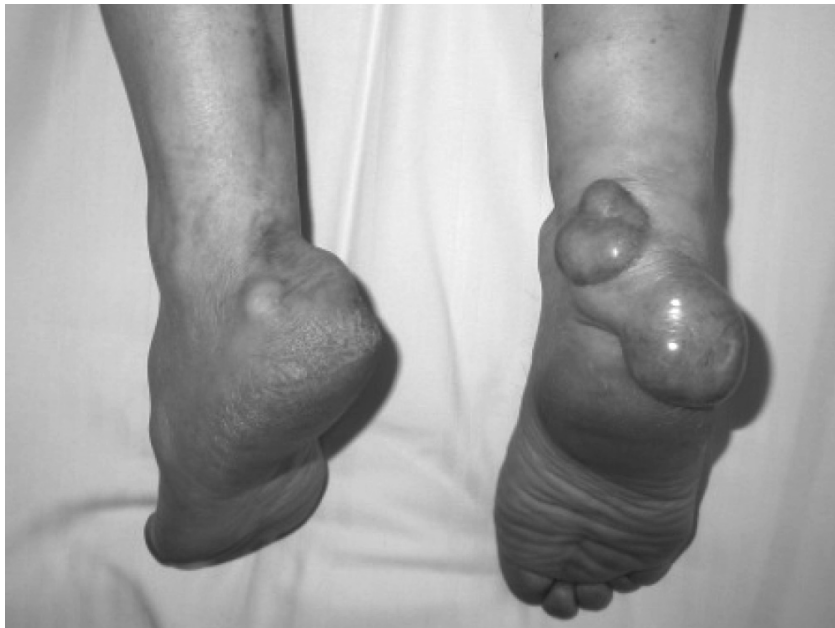

Fig. 1

Photograph showing xanthomatosis nodules in the right tendo Achillis. There are scars from previous surgery for the same problem in the left tendo Achillis which was removed without reconstruction.

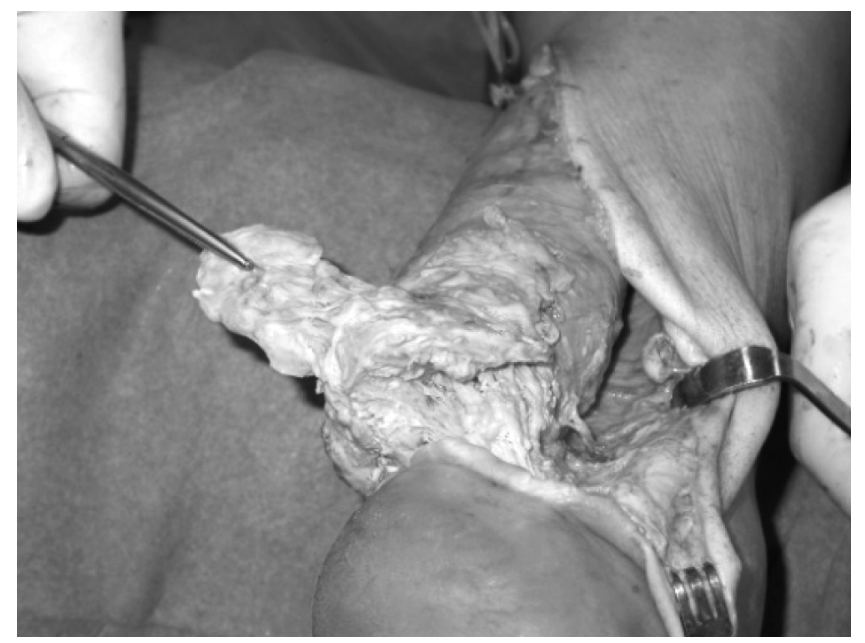

Fig. 3

Photograph showing the tendon to be grossly deformed and thickened with inelastic fibrous tissue replacing the normal tendon.

neum was performed to create a space for the bony component of the graft. After shaping, this was fixed to the calcaneum by two AO partially-threaded $4.5 \mathrm{~mm}$ cancellous screws. The proximal tendinous part of the graft was then sutured end-to-end to the distal part of the triceps surae (Fig. 4). Skin closure was achieved without tension. Healing of the skin was delayed for 35 days because of a small necrotic area in the distal part of the scar. However, this did not need surgical treatment.

The ankle was immobilised non-weight-bearing in a plaster cast at $15^{\circ}$ of plantar flexion for 40 days and then

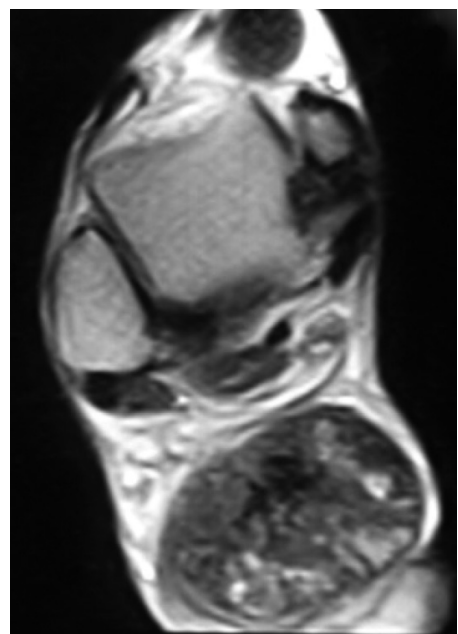

Fig. 2

Pre-operative MRI scan showed a complete alteration of the structure of the tendon and the surrounding soft tissues.

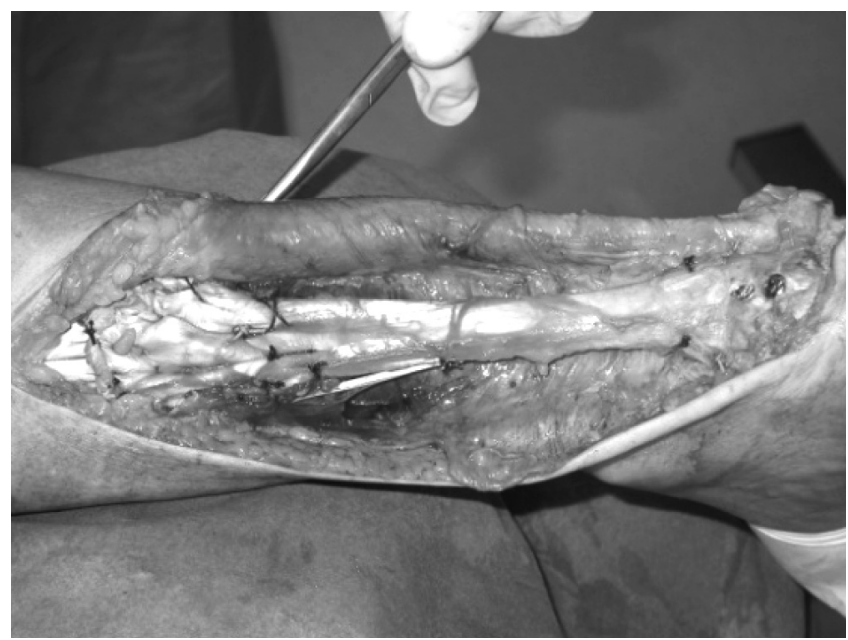

Fig. 4

Photograph showing proximal and distal fixation of the cadaver bonetendon graft.

in a plantigrade cast for 20 days. This cast was removed 60 days post-operatively when active physiotherapy and partial weight-bearing with a heel raise of $3 \mathrm{~cm}$ was allowed.

After satisfactory radiographs had been obtained at 90 days, he was allowed to increase weight-bearing progressively within the limits of comfort. The range of movement at the ankle after removal of the plaster cast 60 days post-operatively was satisfactory. At three months he could walk with one crutch without pain and at four months was able to walk without crutches. At the last 


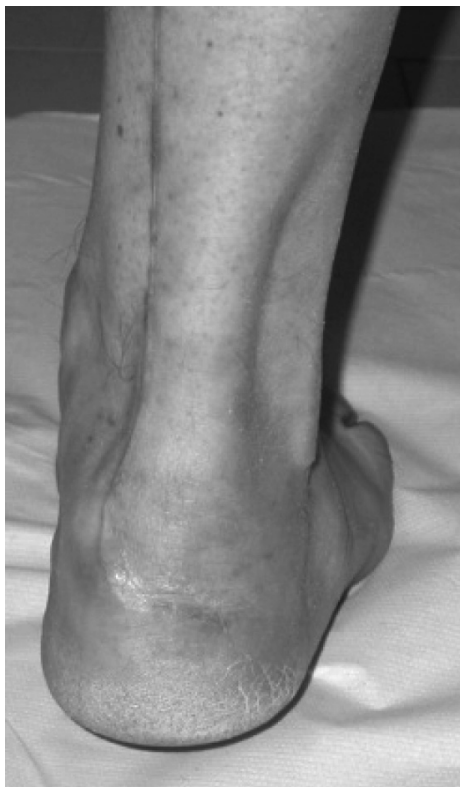

Fig. 3

Photograph eight months postoperatively showing satisfactory healing of the scar and the cosmetic appearance of the tendo Achillis and the heel.

review, eight months after surgery, the result was good, with an American Orthopaedic Foot and Ankle Society (AOFAS) ankle-hindfoot score ${ }^{8}$ of 95/100. Power had not been restored completely, but he could perform a single stance heel raise on the affected side. The subjective cosmetic outcome was satisfactory. The shape of the heel and tendo Achillis had been restored and the scar had healed without fibrosis (Fig. 5). He could wear any type of shoe without problems and his evaluation of the result was very good. He had no symptoms from the region of the graft or the plantar aspect of the heel, despite the protruding screw, seen on plain radiography. There was satisfactory healing of the bony part of the graft (Fig. 6).

\section{Discussion}

A number of procedures have been described for the treatment of chronic degeneration of the tendo Achillis. ${ }^{4-7}$ In our patient we decided to undertake reconstruction using a frozen cadaver allograft because the involvement of the tissues around the tendon was extensive and we were not sure whether other local tendons such as FHL and peroneus brevis were free from disease. The other leg was already compromised functionally because of previous excision of the tendo Achillis without reconstruction. We attempted to find a solution which respected the local anatomy and did not risk morbidity of the local donor site. We preferred the cadaver allograft to a free autolo-

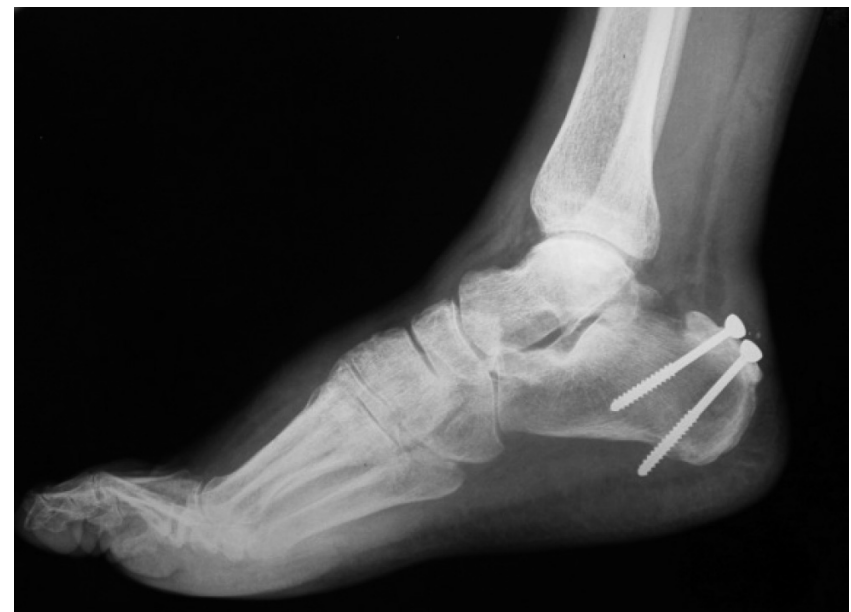

Fig. 4

Plain lateral radiograph of the right foot showing satisfactory bony union of the bone-tendon allograft. The posterior cancellous screw is excessively long and protrudes into the soft tissues of the heel.

gous graft because we thought that fixation would be easier and safer, and the graft stronger.

The use of this type of graft is uncommon in our practice but the tissue bank of the CTO Hospital in Turin is a very satisfactory source of cadaver grafts. One of the screws in the calcaneum was excessively long (Fig. 6), but so far the patient has been free from symptoms in the plantar aspect of the heel. The screw could easily be removed as the bone graft is well healed.

The contralateral tendo Achillis was removed without reconstruction 17 years earlier. The present AOFAS ankle-hindfoot score ${ }^{8}$ for this side is $55 / 100$, because of considerable loss of function and moderate pain in the plantar region of the foot. The cosmetic outcome is unsatisfactory, because of the abnormal appearance and shape of the posterior aspect of the lower leg (Fig. 1). Shoe fitting is also a problem.

Although the follow-up of eight months is short, the early results of the use of a cadaver bone-tendon graft after excision of the tendo Achillis for xanthomatosis are very encouraging, particularly when compared with the simple excision without reconstruction carried out previously on the other side.

No benefits in any form have been received or will be received from a commercial party related directly or indirectly to the subject of this article.

\section{References}

1. Carranza-Bencano A, Fernandez-Centeno M. Xanthomas of the Achilles tendon: report of a bilateral case and a review of literature. Foot Ankle Int 1999;20:314-16.

2. Smithard A, Lamyman MJ, McCarthy CL, et al. Cerebrotendinous xanthomatosis presenting with bilateral Achilles tendon xanthomata. Skeletal Radiol2007;36:171-5.

3. Brodsky JW, Beischer AD, Anat D, et al. Cerebrotendinous xanthomatosis: a rare cause of bilateral Achilles tendon swelling and ataxia: a case report. J Bone Joint Surg [Am] 2006;88-A:1340-4 
4. Pearce CJ, Sexton S, Gerrard D, Hatrick A, Solan M. Successful treatment of a chronically infected and necrotic tendo Achillis in a diabetic with excision, flexor hallucis longus transfer and split-skin grafting. J Bone Joint Surg [Br] 2008;90B:186-8.

5. Gallant GG, Massie C, Turco VJ. Assessment of eversion and plantar flexion strength after repair of Achilles tendon rupture using peroneus brevis tendon transfer. Am J Orthop 1995:24:257-61.
6. Boopalan PR, Jepegnanam TS, Titus VT, Prasad SY, Chittaranjan SB. Open infected Achilles tendon injury: reconstruction of tendon with fascia lata graft and soft tissue cover with a reverse flow sural flap. Foot Ankle Surg 2008;14:96-9.

7. Lepow GM, Green JB. Reconstruction of a neglected Achilles tendon rupture with an Achilles tendon allograft: a case report. J Foot Ankle Surg 2006;45:351-5.

8. Kitaoka HB, Alexander IJ, Adelaar RS, et al. Clinical rating systems for the ankle-hindfoot, midfoot, hallux and lesser toes. Foot Ankle Int 1994;15:349-53. 